\title{
Fractional part integral representation for derivatives of a function related to $\ln \Gamma(x+1)$
}

\author{
Mark W. Coffey \\ Department of Physics \\ Colorado School of Mines \\ Golden, CO 80401
}

(Received

2010)

August 14, 2011

For $0 \neq x>-1$ let

\section{Abstract}

$$
\Delta(x)=\frac{\ln \Gamma(x+1)}{x} .
$$

Recently Adell and Alzer proved the complete monotonicity of $\Delta^{\prime}$ on $(-1, \infty)$ by giving an integral representation of $(-1)^{n} \Delta^{(n+1)}(x)$ in terms of the Hurwitz zeta function $\zeta(s, a)$. We reprove this integral representation in different ways, and then re-express it in terms of fractional part integrals. Special cases then have explicit evaluations. Other relations for $\Delta^{(n+1)}(x)$ are presented, including its leading asymptotic form as $x \rightarrow \infty$.

\section{Key words and phrases}

Gamma function, digamma function, polygamma function, Hurwitz zeta function, Riemann zeta function, fractional part, integral representation

33B15, 11M35, 11Y60

2010 AMS codes 


\section{Introduction and statement of results}

For $0 \neq x>-1$ let

$$
\Delta(x)=\frac{\ln \Gamma(x+1)}{x}, \quad \Delta(0)=-\gamma
$$

where $\Gamma$ is the Gamma function, $\gamma=-\psi(1)$ is the Euler constant, and $\psi(x)=\Gamma^{\prime} / \Gamma$ is the digamma function. The study of the convexity and monotonicity of the functions $\Gamma$ and $\Delta$ and of their derivatives is of interest [8, 13, 14, 17]. For instance, the paper [8] gave an analog of the well known Bohr-Mollerup theorem for the function $\Delta(x)$. Monotonicity and convexity are very useful properties for developing a variety of inequalities. Completely monotonic functions have applications in several branches, including complex analysis, potential theory, number theory, and probability (e.g., [5]). In [4], $-\Delta(x)$ was shown to be a Pick function, with integral representation

$$
-\Delta(x)=-\frac{\pi}{4}+\int_{-\infty}^{-1}\left(\frac{1}{t-z}-\frac{t}{t^{2}+1}\right) \frac{d t}{-t}
$$

I.e., this function is holomorphic in the upper half plane with nonnegative imaginary part.

Recently Adell and Alzer [2] proved the complete monotonicity of $\Delta^{\prime}$ on $(-1, \infty)$ by demonstrating the following integral representation.

Proposition 1. (Adell and Alzer). For $x>-1$ and $n \geq 0$ an integer one has

$$
(-1)^{n} \Delta^{(n+1)}(x)=(n+1) ! \int_{0}^{1} u^{n+1} \zeta(n+2, x u+1) d u
$$

where $\zeta(s, a)$ is the Hurwitz zeta function (e.g., [10]). The complete monotonicity of $\Delta^{\prime}$, the statement $(-1)^{n} \Delta^{(n+1)}(x) \geq 0$, then follows from $\zeta(n+2, x u+1) \geq 0$ for 
$x>-1$. We reprove the result (1.2) in two other ways, and in so doing illustrate properties of the $\zeta$ function.

Corollary 1 . We have the following recurrence:

$$
\frac{(-1)^{n}}{(n+1) !} \Delta^{(n+1)}(x)=\frac{1}{x} \frac{(-1)^{n-1}}{n !} \Delta^{(n)}(x)-\frac{\zeta(n+1, x+1)}{(n+1) x} .
$$

We then relate cases of (1.2) to fractional part integrals, including the following, wherein we let $\{x\}=x-[x]$ denote the fractional part of $x$.

Proposition 2. Let $k \geq 1$ be an integer. Then we have

$$
\int_{0}^{1} u^{n+1} \zeta(n+2, k u+1) d u=\frac{1}{k^{n+2}}\left[\int_{1}^{\infty} \frac{\{w\}^{n+1}}{w^{n+2}} d w+\sum_{j=1}^{k-1} \int_{0}^{\infty} \frac{(\{x\}+j)^{n+1}}{(x+j+1)^{n+2}} d x\right] .
$$

As a further special case we have

Corollary 2. We have

$$
\begin{gathered}
(-1)^{n} \Delta^{(n+1)}(1)=(n+1) ! \int_{0}^{1} y^{n+1} \zeta(n+2, y+1) d y=(n+1) ! \int_{0}^{\infty} \frac{\{x\}^{n+1}}{(x+1)^{n+2}} d x \\
=(n+1) !\left[1-\gamma-\sum_{j=2}^{k-1} \frac{1}{j}[\zeta(j)-1]\right]
\end{gathered}
$$

where $\zeta(s)=\zeta(s, 1)$ is the Riemann zeta function [7, 10, 15, 16].

More generally, we have the following, wherein we put $P_{1}(x)=\{x\}-1 / 2$. Let ${ }_{2} F_{1}$ be the Gauss hypergeometric function [3, 9].

Proposition 3. We have for integers $n \geq 0$

$$
\int_{0}^{1} u^{n+1} \zeta(n+2, x u+1) d u=\frac{1}{2(n+2)} \frac{1}{(x+1)^{n+2}}{ }_{2} F_{1}\left(1, n+2 ; n+3 ; \frac{x}{x+1}\right)
$$


$+\frac{1}{(n+1)(n+2)} \frac{1}{(x+1)^{n+1}}{ }_{2} F_{1}\left(1, n+1 ; n+3 ; \frac{x}{x+1}\right)-\int_{0}^{\infty} \frac{1}{(t+1)} \frac{P_{1}(t)}{(t+x+1)^{n+2}} d t$.

From this Proposition we may then determine the following asymptotic form:

Corollary 3. We have

$$
\Delta^{(n+1)}(x) \sim(-1)^{n} \frac{n !}{(x+1)^{n+1}}, \quad x \rightarrow \infty,
$$

in agreement with Corollary 1.2 of [2]. In fact, the proof shows how higher order terms may be systematically found.

Many expressions may be found for the ${ }_{2} F_{1}$ functions in (1.6) and (2.20) below, and we present a sample of these in an Appendix.

A simple property of $\Delta$ is given in the following.

Proposition 4. We have (a)

$$
\int_{0}^{1} \Delta(x) d x=-\gamma+\sum_{k=2}^{\infty} \frac{(-1)^{k}}{k^{2}} \zeta(k),
$$

and (b)

$$
\int_{0}^{1} \Delta^{2}(x) d x=\gamma^{2}-2 \gamma \sum_{k=2}^{\infty} \frac{(-1)^{k}}{k^{2}} \zeta(k)+\sum_{m=4}^{\infty} \frac{(-1)^{m}}{(m-1)} \sum_{\ell=2}^{m-2} \frac{\zeta(m-\ell) \zeta(\ell)}{(m-\ell) \ell} .
$$

Throughout we let $\psi^{(j)}$ denote the polygamma functions (e.g., [1]), and we note the relation for integers $n>0$

$$
\psi^{(n)}(x)=(-1)^{n+1} n ! \zeta(n+1, x) .
$$

Therefore, as to be expected, (1.2) could equally well be written as an integral over $\psi^{(n+1)}(x u+1)$. The polygamma functions possess the functional equation

$$
\psi^{(j)}(x+1)=\psi^{(j)}(x)+(-1)^{j} \frac{j !}{x^{j+1}} .
$$


For a very recent development of single- and double-integral and series representations for the Gamma, digamma, and polygamma functions, [6] may be consulted.

\section{Proof of Propositions}

Proposition 1. We provide two alternative proofs of this result. The result holds for $n=0$, and for the first proof we proceed by induction. For the inductive step we have

$$
\begin{gathered}
\Delta^{(n+2)}(x)=\frac{d}{d x} \Delta^{(n+1)}(x) \\
=(-1)^{n}(n+1) ! \int_{0}^{1} u^{n+1} \frac{d}{d x} \zeta(n+2, x u+1) d u \\
=(-1)^{n+1}(n+2) ! \int_{0}^{1} u^{n+2} \zeta(n+3, x u+1) d u .
\end{gathered}
$$

In the last step, we used $\partial_{a} \zeta(s, a)=-s \zeta(s+1, a)$.

We remark that this first method shows that (1.2) may be evaluated by repeated integration by parts, for we have

$$
(n+1) ! \int_{0}^{1} u^{n+1} \zeta(n+2, x u+1) d u=\frac{(-1)^{n}}{x^{n}} \int_{0}^{1} u^{n+1}\left(\frac{\partial}{\partial u}\right)^{n} \zeta(2, x u+1) d u .
$$

Second method. By the product rule we have

$$
\begin{gathered}
\Delta^{(n+1)}(x)=\sum_{j=0}^{n+1}\left(\begin{array}{c}
n \\
j
\end{array}\right)[\ln \Gamma(x+1)]^{(n-j)} \frac{(-1)^{j} j !}{x^{j+1}} \\
=\sum_{j=0}^{n+1}\left(\begin{array}{c}
n+1 \\
j
\end{array}\right) \psi^{(n-j)}(x+1) \frac{(-1)^{j} j !}{x^{j+1}} .
\end{gathered}
$$

Here, it is understood that $\psi^{(-1)}(x)=\ln \Gamma(x)$. We now apply (1.9) and the integral representation

$$
(n-j) ! \zeta(n-j+1, x+1)=\int_{0}^{\infty} \frac{t^{n-j} e^{-x t}}{e^{t}-1} d t,
$$


so that

$$
\begin{gathered}
\Delta^{(n+1)}(x)=(-1)^{n+1} \sum_{j=0}^{n+1} \frac{j !}{x^{j+1}} \int_{0}^{\infty} \frac{t^{n-j} e^{-x t}}{e^{t}-1} d t \\
=\frac{(-1)^{n+1}}{x^{n+2}} \int_{0}^{\infty} \frac{e^{-x t}}{\left(e^{t}-1\right)}\left[e^{x t} \Gamma(n+2, x t)-(n+1) !\right] \frac{d t}{t},
\end{gathered}
$$

where the incomplete Gamma function $\Gamma(x, y)$ has the property [9] (p. 941)

$$
\Gamma(n+1, x)=n ! e^{-x} \sum_{m=0}^{n} \frac{x^{m}}{m !}
$$

Now we use a Laplace transform,

$$
\int_{0}^{1} u^{n+1} e^{-x u t} d u=\frac{1}{(x t)^{n+2}}[(n+1) !-\Gamma(n+2, x t)]
$$

to write

$$
\begin{aligned}
\Delta^{(n+1)}(x) & =(-1)^{n} \int_{0}^{\infty} \frac{e^{-x t}}{\left(e^{t}-1\right)} t^{n+1} \int_{0}^{1} u^{n+1} e^{-x t u} d u d t \\
= & (-1)^{n} \int_{0}^{1} u^{n+1} \int_{0}^{\infty} \frac{t^{n+1}}{e^{t}-1} e^{-x u t} d t d u \\
= & -(-1)^{n} \int_{0}^{1} u^{n+1} \zeta(n+2, x u+1) d u
\end{aligned}
$$

By absolute convergence and the Tonelli-Hobson theorem, the interchange of integrations is justified. In the last step, we applied the representation (2.4).

Corollary 1. This is proved by integrating by parts in (1.2).

Remark. It is possible to find explicit expressions for the values $\Delta^{(n+1)}(j+1 / 2)$ with half-integer argument. This is due to the functional equation (1.10) along with the values $\psi^{(-1)}(1 / 2)=\ln \sqrt{\pi}, \psi(1 / 2)=-\gamma-2 \ln 2$, and [1] (p. 260)

$$
\psi^{(n)}\left(\frac{1}{2}\right)=(-1)^{n+1} n !\left(2^{n+1}-1\right) \zeta(n+1), \quad n \geq 1 .
$$


We then obtain, for instance, by using (2.3)

$\frac{\Delta^{(n+1)}\left(-\frac{1}{2}\right)}{(n+1) !}=\sum_{j=0}^{n-1} \frac{(-1)^{n-j}}{(n-j+1)}\left(2^{n+2}-2^{j+1}\right) \zeta(n-j+1)+2^{n+1}(\gamma+2 \ln 2)-2^{n+2} \ln \sqrt{\pi}$

Similarly, it is possible to find explicit expressions for the values $\Delta^{(n+1)}(j+1 / 4)$ and $\Delta^{(n+1)}(j+3 / 4)$ by using the corresponding values of $\psi^{(k)}[11]$.

Proposition 2. We use two Lemmas.

Lemma 1. When the integrals involved are convergent, we have for integrable functions $f$ and $g$

$$
\int_{1}^{\infty} f(\{x\}) g(x) d x=\int_{0}^{1} f(y) \sum_{\ell=1}^{\infty} g(y+\ell) d y
$$

Lemma 2. For $b>0, \lambda>1$, and $c \geq 0$ we have for integrable functions $f$

$$
\int_{0}^{\infty} f\left(\left\{\frac{x}{b}\right\}\right) \frac{d x}{(x+c)^{\lambda}}=\frac{1}{b^{\lambda-1}} \int_{0}^{1} f(y) \zeta(\lambda, y+c / b) d y
$$

This holds when the integrals are convergent.

Proof. For Lemma 1 we have

$$
\begin{aligned}
& \int_{1}^{\infty} f(\{x\}) g(x) d x=\sum_{\ell=1}^{\infty} \int_{\ell}^{\ell+1} f(\{x\}) g(x) d x \\
= & \sum_{\ell=1}^{\infty} \int_{\ell}^{\ell+1} f(x-\ell) g(x) d x=\sum_{\ell=1}^{\infty} \int_{0}^{1} f(y) g(y+\ell) d y .
\end{aligned}
$$

For Lemma 2 we first have

$$
\int_{0}^{\infty} f\left(\left\{\frac{x}{b}\right\}\right) g(x) d x=b \int_{0}^{\infty} f(\{v\}) g(b v) d v
$$




$$
\begin{aligned}
& =b \sum_{\ell=0}^{\infty} \int_{\ell}^{\ell+1} f(v-\ell) g(b v) d v \\
& =b \sum_{\ell=0}^{\infty} \int_{0}^{1} f(y) g[b(y+\ell)] d y .
\end{aligned}
$$

We now put $g(x)=1 /(x+c)^{\lambda}$, so that

$$
\sum_{\ell=0}^{\infty} g[b(y+\ell)]=\frac{1}{b^{\lambda}} \sum_{\ell=0}^{\infty} \frac{1}{(y+\ell+c / b)^{\lambda}}=\frac{1}{b^{\lambda}} \zeta(\lambda, y+c / b) .
$$

Proof of Proposition 2. We have for integers $k \geq 1$

$$
\begin{gathered}
\int_{0}^{1} u^{n+1} \zeta(n+2, k u+1) d u=\frac{1}{k^{n+2}} \int_{0}^{k} v^{n+1} \zeta(n+2, v+1) d v \\
=\frac{1}{k^{n+2}} \sum_{\ell=0}^{k-1} \int_{\ell}^{\ell+1} v^{n+1} \zeta(n+2, v+1) d v \\
=\frac{1}{k^{n+2}} \sum_{\ell=0}^{k-1} \int_{0}^{1}(w+\ell)^{n+1} \zeta(n+2, w+\ell+1) d v .
\end{gathered}
$$

We now apply Lemma 2 with $b=1, c=\ell+1$, and $f(w)=(w+\ell)^{n+1}$, giving

$$
\begin{gathered}
\int_{0}^{1} u^{n+1} \zeta(n+2, k u+1) d u=\frac{1}{k^{n+2}} \sum_{\ell=0}^{k-1} \int_{0}^{\infty} \frac{(\{x\}+\ell)^{n+1}}{(x+\ell+1)^{n+2}} d x \\
=\frac{1}{k^{n+2}}\left[\int_{1}^{\infty} \frac{\{w\}^{n+1}}{w^{n+2}} d w+\sum_{\ell=1}^{k-1} \int_{0}^{\infty} \frac{(\{x\}+\ell)^{n+1}}{(x+\ell+1)^{n+2}} d x\right] .
\end{gathered}
$$

In the last step we used the periodicity $\{w-1\}=\{w\}$.

For Corollary 2, we apply Lemma 2 of [6].

Proposition 3. We start from the integral representation

$$
\zeta(s, a)=\frac{a^{-s}}{2}+\frac{a^{1-s}}{s-1}-s \int_{0}^{\infty} \frac{P_{1}(x)}{(x+a)^{s+1}} d x, \quad \text { Re } s>-1 .
$$

Then

$$
\int_{0}^{1} u^{n+1} \zeta(n+2, x u+1) d u=\int_{0}^{1} u^{n+1}\left[\frac{1}{2(x u+1)^{n+2}}+\frac{1}{(n+1)(x u+1)^{n+1}}\right.
$$




$$
\left.-(n+2) \int_{0}^{\infty} \frac{P_{1}(t) d t}{(t+x u+1)^{n+3}}\right] d u .
$$

By using a standard integral representation of ${ }_{2} F_{1}$ (e.g., [9], p. 1040 or [3] p. 65) we have

$$
\begin{gathered}
\int_{0}^{1} u^{n+1} \zeta(n+2, x u+1) d u=\frac{1}{2(n+2)}{ }_{2} F_{1}(n+2, n+2 ; n+3 ;-x) \\
+\frac{1}{(n+1)(n+2)}{ }_{2} F_{1}(n+1, n+2 ; n+3 ;-x)-\int_{0}^{\infty} \frac{1}{(t+1)} \frac{P_{1}(t)}{(t+x+1)^{n+2}} d t .
\end{gathered}
$$

By applying a standard transformation rule [9] (p. 1043) to the ${ }_{2} F_{1}$ functions, we obtain the Proposition.

Corollary 3. We give the detailed asymptotic forms as $x \rightarrow \infty$ of the hypergeometric functions in (1.6). We easily have that ${ }_{2} F_{1}(1, n+1 ; n+3 ; 1)=n+2$ and these forms will then show that the corresponding term in (1.6) gives the leading term as $x \rightarrow \infty$. We let $(a)_{j}=\Gamma(a+j) / \Gamma(a)$ be the Pochhammer symbol. The following expansions are valid for $|z-1|<1$ and $|\arg (1-z)|<\pi$ :

$$
{ }_{2} F_{1}(1, y ; 1+y ; z)=y \sum_{k=0}^{\infty} \frac{(y)_{k}}{k !}[\psi(k+1)-\psi(k+y)-\ln (1-z)](1-z)^{k},
$$

and

${ }_{2} F_{1}(1, y ; 2+y ; z)=y+1-y(y+1) \sum_{k=0}^{\infty} \frac{(y+1)}{k !}[\psi(k+1)-\psi(k+y+1)-\ln (1-z)](1-z)^{k+1}$,

where $(y)_{0}=1$. These expansions are the $n=0$ and $n=1$ cases of (9.7.5) in [12] (p. $257)$, respectively. We put $y=n+1, z=x /(x+1), \ln (1-z)=-\ln (x+1)$ and then 
find

$$
{ }_{2} F_{1}\left(1, n+1 ; n+3 ; \frac{x}{x+1}\right)=n+2+(n+1)(n+2)[\gamma-\ln x+\psi(n+2)] \frac{1}{x}+O\left(\frac{\ln x}{x^{2}}\right),
$$

and

$$
{ }_{2} F_{1}\left(1, n+2 ; n+3 ; \frac{x}{x+1}\right)=-(n+2)[\gamma-\ln x+\psi(n+2)]+O\left(\frac{\ln x}{x}\right) .
$$

The integral term in (1.6) is at most $O\left[(x+1)^{-(n+2)}\right]$, and is actually much smaller due to cancellation within the integrand, and the Corollary then follows.

Remarks. Of course we have from $(1.2) \Delta^{(n+1)}(0)=(-1)^{n}(n+1) ! \zeta(n+2) /(n+2)$, in agreement with the expansion (2.26). This special case is recovered from Proposition 3 in the following way. We have ${ }_{2} F_{1}(a, b ; c ; 0)=1$ and the representation [16] (p. 14)

$$
\zeta(s)=\frac{1}{s-1}+\frac{1}{2}-s \int_{1}^{\infty} \frac{P_{1}(x)}{x^{s+1}} d x,
$$

the $a=1$ case of (2.18), giving the identity $\int_{0}^{1} u^{n+1} \zeta(n+2,1) d u=\zeta(n+2) /(n+2)$.

In connection with Propositions 2 and 3, another representation that might be employed is [7]

$$
\ln \Gamma(x+1)=\left(x+\frac{1}{2}\right) \ln x-x+\frac{1}{2} \ln 2 \pi-\int_{0}^{\infty} \frac{P_{1}(t)}{t+x} d t .
$$

We may note that representations (2.19) or (2.20), for instance, provide another basis for proving integral representations for $(-1)^{n} \Delta^{(n+1)}(x)$ by induction. When using (2.20), we use the derivative property

$$
\frac{d}{d x}{ }_{2} F_{1}(a, b ; c ;-x)=-\frac{a b}{c}{ }_{2} F_{1}(a+1, b+1 ; c+1 ;-x) .
$$


The ${ }_{2} F_{1}$ function in (1.6) can be written in other ways, including using a transformation formula [9] (p. 1043), so that

$$
{ }_{2} F_{1}\left(1, n+2 ; n+3 ; \frac{x}{x+1}\right)=(x+1){ }_{2} F_{1}(1,2 ; n+3 ;-x) .
$$

Proposition 4. The result uses the expansion [9] (p. 939)

$$
\ln \Gamma(x+1)=-\gamma x+\sum_{k=2}^{\infty} \frac{(-1)^{k}}{k} \zeta(k) x^{k} .
$$

Remark. Let $\operatorname{Ei}(x)$ be the exponential integral function (e.g., 9], p. 925). Given the relations [9] (pp. 927, 942)

$$
\Gamma(0, x)=-\operatorname{Ei}(-x)=-\left(\gamma+\ln x+\sum_{k=1}^{\infty} \frac{(-x)^{k}}{k k !}\right),
$$

it is possible to write

$$
\int_{0}^{1} \Delta(x) d x=-\gamma-\int_{0}^{\infty} \frac{[\gamma-t+\Gamma(0, t)+\ln t]}{t\left(e^{t}-1\right)} d t .
$$

This follows by inserting a standard integral representation for the values $\zeta(k)$ into the right side of (1.8a).

\section{Acknowledgement}

I thank J. A. Adell for reading the manuscript. 


\section{Appendix}

Here we present illustrative relations for the sort of hypergeometric functions appearing in (1.6) and (2.20).

The contiguous relations [9] (pp. 1044-45) may be readily applied. As well, we have for instance [9] (p. 1043)

$$
{ }_{2} F_{1}(n+2, n+2 ; n+3 ;-x)=(1+x)^{-(n+1)}{ }_{2} F_{1}(1,1 ; n+3 ;-x) .
$$

The next result provides a type of recurrence relation in the first parameter of the ${ }_{2} F_{1}$ function.

Proposition A1. For integers $n \geq-1$ we have

$$
\begin{aligned}
& \int_{0}^{1} \frac{u^{n+1}}{(x u+1)^{n+2}} d u=\frac{1}{(n+2)}{ }_{2} F_{1}(n+2, n+2 ; n+3 ;-x) \\
= & \frac{1}{(n+1)}\left[\frac{1}{(x+1)^{n+1}}-\frac{1}{(n+2)}{ }_{2} F_{1}(n+1, n+2 ; n+3 ;-x)\right] .
\end{aligned}
$$

Proof. With $v=x u$ in (A.2), we have

$$
\begin{gathered}
\frac{1}{x^{n+2}} \int_{0}^{x} \frac{v^{n+1}}{(v+1)^{n+2}} d v=\frac{1}{x^{n+2}} \int_{0}^{x}[(v+1)-v] \frac{v^{n+1}}{(v+1)^{n+2}} d v \\
=\frac{1}{x^{n+2}}\left[\int_{0}^{x} \frac{v^{n+1}}{(v+1)^{n+1}} d v-\int_{0}^{x} \frac{v^{n+2}}{(v+1)^{n+2}} d v\right] \\
=\frac{1}{x^{n+2}}\left[\int_{0}^{x} \frac{v^{n+1}}{(v+1)^{n+1}} d v-\frac{(n+2)}{(n+1)} \int_{0}^{x} \frac{v^{n+1}}{(v+1)^{n+1}} d v+\frac{1}{(n+1)} \frac{x^{n+2}}{(x+1)^{n+1}}\right],
\end{gathered}
$$

where we integrated by parts. Using a standard integral representation for ${ }_{2} F_{1}[9]$ (p. 1040) leads to the Proposition. 
Proposition A1 may be iterated in the first parameter of the ${ }_{2} F_{1}$ function. Then the following relation may be applied:

$$
\int_{0}^{1} \frac{u^{n+1}}{(x u+1)^{2}} d u=\frac{1}{1+x}-\left(\frac{n+1}{n+2}\right){ }_{2} F_{1}(1, n+2 ; n+3 ;-x) .
$$

The ${ }_{2} F_{1}$ functions of concern here may be written with one or more terms containing $\ln (x+1)$. One way to see this is the following. We have for the function of (A.4), first integrating by parts,

$$
\begin{gathered}
\int_{0}^{1} \frac{u^{n+1}}{(x u+1)^{2}} d u=-\frac{1}{x}\left[(n+1) \int_{0}^{1} \frac{u^{n}}{x u+1} d u+\frac{1}{x+1}\right] \\
=-\frac{1}{x}\left[\frac{(n+1)}{x^{n+1}} \int_{0}^{x} \frac{v^{n}}{v+1} d v+\frac{1}{x+1}\right] \\
=-\frac{1}{x}\left[\frac{(n+1)}{x^{n+1}} \int_{0}^{x} \frac{\left[1-\left(1-v^{n}\right)\right]}{v+1} d v+\frac{1}{x+1}\right] \\
=-\frac{1}{x}\left\{\frac{(n+1)}{x^{n+1}}\left[\ln (x+1)-\int_{0}^{x} \frac{\left(1-v^{n}\right)}{v+1} d v\right]+\frac{1}{x+1}\right\} .
\end{gathered}
$$

For $0 \leq x \leq 1$ we may note the following simple inequality for the integral of (A.5):

$$
\int_{0}^{x} \frac{\left(1-v^{n}\right)}{v+1} d v \leq \int_{0}^{x}\left(1-v^{n}\right) d v \leq x .
$$




\section{References}

[1] M. Abramowitz and I. A. Stegun, Handbook of Mathematical Functions, Washington, National Bureau of Standards (1964).

[2] J. A. Adell and H. Alzer, A monotonicity property of Euler's gamma function, Publ. Math. Debrecen (2010).

[3] G. E. Andrews, R. Askey, and R. Roy, Special Functions, Cambridge University Press (1999).

[4] C. Berg and H. L. Pedersen, Pick functions related to the gamma function, Rocky Mtn J. Math. 32, 507-525 (2002).

[5] S. Bochner, Harmonic analysis and the theory of probability, Univ. California Press (1955).

[6] M. W. Coffey, Integral and series representations of the digamma and polygamma functions, arXiv:1008.0040v2 (2010).

[7] H. M. Edwards, Riemann's Zeta Function, Academic Press, New York (1974).

[8] P. J. Grabner, R. F. Tichy, and U. T. Zimmerman, Inequalities for the gamma function with applications to permanents, Discrete Math. 154, 53-62 (1996).

[9] I. S. Gradshteyn and I. M. Ryzhik, Table of Integrals, Series, and Products, Academic Press, New York (1980). 
[10] A. Ivić, The Riemann Zeta-Function, Wiley New York (1985).

[11] K. S. Kölbig, The polygamma function $\psi^{(k)}(x)$ for $x=\frac{1}{4}$ and $x=\frac{3}{4}$, J. Comput. Appl. Math. 75, 43-46 (1996).

[12] N. N. Lebedev, Special functions and their applications, Dover Publications (1972).

[13] F. Qi and C.-P. Chen, A complete monotonicity property of the gamma function, J. Math. Analysis Appl. 296, 603-607 (2004).

[14] F. Qi and B.-N. Guo, Some logarithmically completely monotonic functions related to the gamma function, 47, 1283-1297 (2010).

[15] B. Riemann, Über die Anzahl der Primzahlen unter einer gegebenen Grösse, Monats. Preuss. Akad. Wiss., 671 (1859-1860).

[16] E. C. Titchmarsh, The Theory of the Riemann Zeta-Function, 2nd ed., Oxford University Press, Oxford (1986).

[17] H. Vogt and J. Voigt, A monotonicity property of the $\Gamma$-function, J. Inequal. Pure Appl. Math. 3, Art. 73 (2002). 